Revista de Psicología Vol. 39 (2), 2021 (e-ISSN 2223-3733)

\title{
Adaptación argentina de la nueva escala de Orientación a la Dominancia Social $\left(\mathrm{SDO}_{7}\right)$
}

\author{
María Belén Casalá ${ }^{1}$, Gisela Delfino ${ }^{2}$ \\ Pontificia Universidad Católica Argentina ${ }^{1,2}$, CONICET $^{2}$ y Universidad Andrés \\ Bello-Chile ${ }^{2}$
}

El presente trabajo adaptó la séptima escala de Dominancia Social en Argentina en sus dos versiones: larga (16 ítems) y corta (ocho ítems; Ho et al., 2015). Se estudiaron las propiedades psicométricas de la escala y se evaluó su estructura factorial. Se analizan las diferencias en SDO en cuanto a sexo, edad, posicionamiento socioeconómico y orientación política. Se trabajó con una muestra no probabilística intencional $(n=1573$, escala corta; $n=590$, escala larga) entre 18 y 60 años. La escala exhibe niveles de confiabilidad consistentes con la teoría. El análisis factorial confirma la estructura bifactorial de la escala, encontrando dos dimensiones: SDO-Dominante y SDO-E (Anti)Igualitarismo. Se encontró una correlación positiva entre el posicionamiento político de derecha y el SDO.

Palabras clave: Escala de dominancia social, Escala $\mathrm{SDO}_{7}$, jerarquías grupales, desigualdad.

\section{Argentinian adaptation of the new Social Dominance Orientation scale ( $\mathrm{SDO}_{7}$ )}

The present work adapted the seventh scale of Social Dominance in Argentina in its two versions: long (16 items) and short (eight items; Ho et al., 2015). The psychometric properties of the scale were studied and its factorial structure was evaluated. We analyzed differences in SDO as regards sex, age, socioeconomic self-positioning and political orientation. We worked with a convenience sample ( $n=1573$, short scale; $n=590$, long scale) between 18 and 60 years. The scale shows evidence of reliability consistent with the theory. The factor analysis confirms the bifactorial structure of the scale, finding two dimensions: SDO-Dominance and SDO-E Egalitarianism. A positive correlation was found between right-wing political self-positioning and SDO.

Keywords: Social Dominance Scale, $\mathrm{SDO}_{7}$ Scale, group hierarchies, inequality.

1 Licenciada en Psicología, Universidad Católica Argentina, Argentina. Contacto: casalabelen@gmail.com

2 Licenciada y doctora en Psicología, Universidad de Buenos Aires, Argentina. Profesora titular de la Pontificia Universidad Católica Argentina (UCA). Investigadora adjunta del Consejo Nacional de Investigaciones Científicas y Técnicas (CONICET) en el Centro de Investigaciones en Psicología y Psicopedagogía (CIPP) de la UCA. Profesora visitante, doctorado en Educación y Sociedad, Universidad Andrés Bello, Chile. Contacto: gisela_delfino@uca.edu.ar https://orcid.org/0000-0002-3732-184X 


\section{Adaptação Argentina da nova escala de Orientaçáo de Dominância Social ( $\left.\mathrm{SDO}_{7}\right)$}

Este trabalho adaptou a sétima escala de Dominância Social na Argentina em suas duas versóes: longa (16 itens) e curta (oito itens; Ho et al., 2015). As propriedades psicométricas da escala foram estudadas e sua estrutura fatorial foi avaliada. São analisadas as diferenças de SDO em termos de sexo, idade, posicionamento socioeconômico e orientação política. Trabalhamos com uma amostra não probabilística intencional $(\mathrm{N}=1573$, escala curta; $\mathrm{N}$ = 590, escala longa) entre 18 e 60 anos. A escala exibe níveis de confiabilidade consistentes com a teoria. A análise fatorial confirma a estrutura bifatorial da escala, encontrando duas dimensóes: SDO-Dominante e SDO-E (Anti) Equalismo. Foi encontrada uma correlação positiva entre o posicionamento político de direita e o SDO.

Palavras-chave: Escala de dominância social, escala $\mathrm{SDO}_{7}$, hierarquias de grupos, desigualdade.

\section{Adaptation en Argentine de la nouvelle échelle d'Orientation de la Dominance Sociale (SDO7)}

Le présent travail a adapté la septième échelle de la dominance sociale en Argentine dans ses deux versions: longue (16 éléments) et courte (huit éléments; Ho et al., 2015). Les propriétés psychométriques de l'échelle ont été étudiées et sa structure factorielle a été évaluée. Les différences d'SDO en termes de sexe, d'âge, de positionnement socio-économique et d'orientation politique sont analysées. Nous avons travaillé avec un échantillon intentionnel non probabiliste ( $n=1573$, échelle courte; $n=590$, échelle longue) entre 18 et 60 ans. L'échelle présente des niveaux de fiabilité conformes à la théorie. L'analyse factorielle confirme la structure bifactorielle de l'échelle, trouvant deux dimensions: SDO-Dominant et SDO-E (Anti) Equalism. Une corrélation positive a été trouvée entre le positionnement politique de droite et le SDO.

Mots-clés: échelle de dominance sociale, échelle $\mathrm{SDO}_{7}$, hiérarchies de groupes, inégalités. 
La Orientación a la Dominancia Social (Social Dominance Orientation, en adelante SDO) mide la actitud individual hacia la inequidad entre grupos sociales (Pratto, Sidanius, Stallworth \& Malle, 1994). Esta orientación se expresa en actitudes personales de discriminación y participación en procesos institucionales e intergrupales que producen más beneficios sobre aquellos grupos considerados dominantes, por sobre los subordinados o percibidos como inferiores. La SDO sobre los grupos sociales en general, interactúa con las fuerzas sociales e institucionales produciendo y perpetuando la desigualdad social (Pratto, Sidanius \& Levin, 2006; Pratto et al., 1994). Los niveles individuales de SDO predicen y permiten comprender una amplia gama de actitudes y comportamientos, no solo actuales, sino también pasados, dando lugar a que el constructo ocupe un sólido lugar en el centro de la psicología social y política (Lee, Pratto \& Johnson, 2011). La SDO es particularmente útil ya que no se refiere a grupos específicos, sino que es válida interculturalmente (Sidanius, Pratto, Van Laar \& Levin, 2004) y fue probada en múltiples países y lenguas (Pratto et al., 2000).

Con el objetivo de poder evaluar empíricamente el constructo SDO, Pratto y cols. (1994) construyeron dos escalas: una versión de 14 y otra de 16 ítems, que más tarde Sidanius y Pratto (1999) denominaron como $\mathrm{SDO}_{5} \mathrm{y} \mathrm{SDO}_{6}$ respectivamente. Esta última fue la que mejores propiedades psicométricas obtuvo, ya que obtuvo una fiabilidad original con muestras americanas de $\alpha=$,91 (Pratto et al., 1994), siendo la más utilizada hasta la introducción de la $\mathrm{SDO}_{7}$ (Ho et al., 2015). La $\mathrm{SDO}_{5}$ mostró una fiabilidad promedio $(\alpha=, 82)$ en 16 muestras diferentes e independientes con 2150 participantes de cuatro naciones diferentes (Estados Unidos, Canadá, Taiwán y México; Sidanius \& Pratto, 1999). Por otro lado, la $\mathrm{SDO}_{6}$ posee una fiabilidad promedio de $\alpha=, 83$ en 14 estudios independientes con 4827 participantes de seis naciones diferentes (Estados Unidos, Israel, Palestina, China, Nueva Zelanda y Canadá; Pratto, Sidanius \& Levin, 2006). 
Cuando la escala fue desarrollada por Pratto y cols. (1994) se halló que se comportaba unidimensionalmente, y como constructo único, la SDO ha sido muy útil para entender actitudes y comportamientos intergrupales. Sin embargo, análisis factoriales y estudios posteriores sugirieron que los ítems positivos e invertidos de la escala de 16 ítems $\mathrm{SDO}_{6}$ se comportan bifactorialmente (Cárdenas, Meza, Lagues \& Yañez, 2010; Hindriks, Verkuyten, \& Coenders, 2014; Ho et al., 2012; Silván-Ferrero \& Bustillos 2007). Jost y Thompson (2000) propusieron la existencia de dos dimensiones dentro del constructo de la SDO: la Orientación a la Dominancia Grupal y la Oposición a la Igualdad. Para estos autores, si bien ambas dimensiones en su conjunto componen la SDO, la primera alude a una forma de justificación del propio grupo, mientras que la segunda se refiere a una forma de justificación del sistema.

A partir de estos hallazgos, Ho y cols. (2015) desarrollaron una nueva escala de SDO, conocida como $\mathrm{SDO}_{7}$. Esta nueva forma de medición y conceptualización teórica abarca la estructura bifactorial del constructo de la Orientación a la Dominancia Social. Ho y cols. (2012) nombraron a estas dos subdimensiones como la SDO "Dominante" (SDO-D, Dominance) y la SDO "Igualitarismo" (SDO-E, Egalitarianism), denominaciones que se utilizarán en el presente estudio. Esta escala permite predecir actitudes y comportamientos intergrupales con aún más precisión que la $\mathrm{SDO}_{6}$, siendo igualmente predictiva de las variables teóricamente relevantes (Ho et al., 2015).

En las escalas SDO anteriores (Pratto et al., 1994), los mismos elementos que se utilizan para medir la dimensión dominante son aquellos redactados en una dirección positiva (tales que los puntajes altos en estos ítems indican una SDO alta); por el contrario, todos los ítems utilizados para abordar el igualitarismo están redactados en la dirección contraria, es decir, tienen puntaje inverso (de modo que los puntajes altos denotan una SDO baja). Esto representaría una

3 Para conservar la nomenclatura del artículo original (Ho et al., 2015) se la denomina Igualitarismo aunque, en rigor, esta dimensión hace referencia al anti-igualitarismo. 
confusión de las dimensiones de "método" de la escala (la redacción de los ítems positivos e inversos) con sus dimensiones sustantivas (dominante e igualitaria; Ho et al. 2015; Ho et al., 2012; Jost \& Thompson, 2000).

Ho y cols. (2015) desarrollaron esta nueva medida de SDO que evalúa cada subdimensión de manera equilibrada, con la misma cantidad de ítems positivos que invertidos. Como anteriormente se suponía que la SDO era unidimensional, las versiones anteriores de la escala SDO no abordaron este problema. Debido a que tanto las diferencias de contenido como de redacción influyen en cómo responden los participantes (Bishop, Tuchfarber \& Oldendick, 1978), los autores crearon un modelo de cuatro factores: SDO-D, SDO-E, ítems positivos e ítems invertidos, con cada elemento teniendo una dimensión sustantiva (SDO-D o SDO-E) y un método (positivo o invertido). Además, cada dimensión de la nueva escala tiene validez predictiva por sí misma. Mientras que SDO-D es un predictor más fuerte de actitudes y comportamientos intergrupales que contribuyen a una dominación manifiesta, SDO-E predice otros comportamientos y creencias, como el conservadurismo político o la oposición a políticas gubernamentales que promueven una mayor igualdad.

Los autores propusieron una versión larga de la $\mathrm{SDO}_{7}$ de 16 ítems y una corta para cuando las restricciones del espacio y/o tiempo no permiten el uso de la primera (Ho et al., 2015). En la escala corta, cada subdimensión posee cuatro ítems (dos positivos y dos invertidos) que se seleccionaron en función de los análisis factoriales y las regresiones de los 16 elementos de una muestra de la escala completa. Se ha demostrado que las dos versiones poseen propiedades psicométricas similares.

Desde la introducción de la escala SDO (Pratto et al., 1994), se adaptó y validó en distintos contextos culturales. En español, la escala $\mathrm{SDO}_{6}$ ha sido adaptada en Chile (Cárdenas et al., 2009), en España (Silván-Ferrero \& Bustillos, 2007) y en Argentina (Etchezahar, PradoGascó, Jaume \& Brussino, 2014). En el contexto argentino, $\mathrm{SDO}_{6}$ se ha utilizado en estudios anteriores. En estos los resultados sobre dominancia social indican niveles bajos nacionales de SDO en com- 
paración con la media internacional (Achaval, 2011; Beramendi \& Zubieta, 2016; Muratori, Delfino \& Zubieta, 2012; Petit \& Costa, 2011; Zubieta, Beramendi, Sosa \& Torres, 2011; Zubieta, Delfino \& Fernández, 2007).

Con respecto a las adaptaciones anteriores de la $\mathrm{SDO}_{7}$, esta únicamente se ha validado y adaptado en Italia (Aiello, Passini, Tesi, Morselli $\&$ Pratto, 2019) y en Indonesia (Arifianto, 2018). La adaptación de esta escala en nuestro país marca un antecedente para la comprensión de los motivos que subyacen a una amplia gama de actitudes y comportamientos intergrupales, desde la oposición a las políticas sociales que promueven la igualdad hasta violencia genocida hacia los grupos minoritarios.

\section{Teoría de la Dominancia Social (SDT)}

La Teoría de la Dominancia Social (Social Dominance Theory, en adelante SDT) se desarrolla como explicación a la naturaleza del prejuicio y a la opresión intergrupal que forman parte intrínseca de todas las sociedades humanas. Esta parte del supuesto de que en todas las sociedades surge el conflicto intergrupal como una dimensión inevitable de la vida social (Pratto et al., 1994).

La SDT postula que todas las sociedades humanas tienden a estructurarse como sistemas de jerarquías sociales basadas en grupos. Estas consisten en uno o varios grupos hegemónicos y dominantes en la parte superior, y uno o varios grupos subordinados en la inferior. Entre otras cosas, el o los grupos dominantes se caracterizan por poseer valores sociales positivos, o por tener elementos simbólicos y materiales deseables socialmente. En contraste, a los grupos sociales subordinados se les atribuye un exagerado valor social negativo que incluye, por ejemplo, bajo poder y estatus social, por lo cual se enfrentan a discriminación institucional e interpersonal (Sidanius, Liu, Pratto \& Shaw, 1994; Sidanius \& Pratto, 2004).

Las sociedades están compuestas por: (a) edad, en el cual los adultos tienen un poder desproporcionado por sobre los niños y los jóvenes; (b) 
sexo, en donde los hombres tienen un poder político, económico, social y militar desproporcionado en comparación con el de las mujeres; y (c) relaciones intergrupales arbitrarias. Este último se refiere a grupos que se construyen a partir de elementos distintivos de estos, como, por ejemplo: la raza, la religión, la clase social u orientación política (Del Prado \& Bustillos, 2007; Pratto et al., 2000; Pratto et al., 2006; Pratto, Stallworth \& Sidanius, 1997; Sidanius, Levin, Liu \& Pratto, 2000; Sidanius, Liu et al., 1994; Sidanius \& Pratto, 1999, 2004; Sidanius, Pratto \& Brief, 1995; Sidanius et al., 2004).

Partiendo de esta estructuración trimórfica, Sidanius y Pratto (2004) postulan tres principios básicos de la SDT. En primer lugar, las jerarquías fundadas sobre el sexo y la edad inevitablemente están presentes en todo sistema social, y los sistemas jerárquicos arbitrarios nacen invariablemente de los excedentes económicos de las sociedades. El segundo postulado consiste en que la mayoría de los tipos de opresión grupal como lo son el racismo, el sexismo, el nacionalismo, el clasismo, la intolerancia religiosa y la hostilidad hacia los enfermos mentales, son diferentes manifestaciones de la tendencia humana básica a formar en la sociedad jerarquías sociales basadas en grupos. Por último, en toda estructura social hay fuerzas contrapuestas reforzadoras (hierarchy-enhancing, en adelante HE) y atenuantes de la jerarquía (hierarchy-attenuating, en adelante HA).

Todo sistema ideológico posee un consenso social en actitudes, creencias y estereotipos llamados "mitos legitimadores" por medio de los cuales los miembros de la sociedad minimizan el conflicto entre grupos y a la vez, mantienen y justifican su posición grupal (Sidanius \& Pratto, 1999; Sidanius, Pratto, Martin \& Stallworth, 1991). Se pueden diferenciar dos tipos: por un lado, las ideologías que proveen apoyo hacia la igualdad grupal y social se conceptualizan como mitos legitimadores HA. Estos favorecen las ideologías que problematizan la desigualdad social existente, como la creencia de que la salud, la educación o los salarios dignos son derechos humanos universales (Pratto et al., 2006). Por el otro lado, los mitos HE, acentúan la jerarquía entre grupos al legitimar moral e intelectualmente la desigualdad. Entre estas 
ideologías se hallan el sexismo, el racismo, el karma, la ética protestante, el nacionalismo, entre otros (Kteily, Sheehy-Skeffington \& Ho, 2016; Sidanius \& Pratto, 1999).

Las ideologías HE se comparten en gran parte de manera consensuada en la sociedad. Estas creencias coordinan el comportamiento jerárquico entre instituciones y personas de diferentes grupos sociales, e ideológicamente permiten la reproducción continua de la desigualdad, resultando en una discriminación intergrupal sistemática (Sidanius, Pratto \& Bobo, 1996; Sidanius \& Pratto, 1999). Se encontró una necesidad social y psicológica de aceptar el status quo de la sociedad jerárquica no solo en los grupos de mayor estatus, sino también en aquellos grupos que están en desventaja (Jost \& Banaji, 1994). Sin embargo, aunque los miembros de los grupos subordinados aceptan los mitos legitimadores de la jerarquía, los miembros de los grupos dominantes los aceptan en mayor grado, y, por tanto, tienen mayor SDO (Sidanius, Pratto et al., 1996). Este fenómeno se conoce como asimetría ideológica, uno de los tipos de asimetría comportamental, uno de los elementos centrales de la SDT. Hace referencia a las diferencias en el comportamiento de los individuos pertenecientes a distintos grupos del sistema jerárquico. Esta asimetría refuerza la jerarquía por medio de patrones de socialización, estereotipos e ideologías HE, y pone el acento en cómo las personas pertenecientes a los grupos desfavorecidos participan y contribuyen a su propia subordinación. Esto no implica que no se resistan a la discriminación y opresión, sino que varias de las acciones que llevan a cabo favorecen el sistema de desigualdad beneficiando a los grupos dominantes (Silván-Ferrero \& Bustillos, 2007).

\section{Orientación a la Dominancia Social (SDO)}

Universalmente, todas las personas ejercen y expresan cuatro tipos básicos de relaciones sociales, uno de ellos es el Authority Ranking. En las relaciones asimétricas, las personas se ordenan de manera lineal en las jerarquías sociales. Mientras que aquellos individuos con rango superior poseen más autoridad, prestigio y privilegios, los subordinados tienen derecho a orientación y protección. Así, aunque la SDO es una 
manifestación de Authority Ranking, no todo el apoyo a la jerarquía es SDO (Fiske, 1992). Las estructuras que mantienen la desigualdad entre grupos sociales se ven reforzadas por los mitos HE. La aceptación de estos está en parte determinada por el mecanismo psicológico individual de la SDO, caracterizada como la predisposición individual general hacia las relaciones intergrupales jerárquicas y no igualitarias. Esta interactúa con fuerzas sociales e institucionales para producir y reproducir la inequidad social (Pratto et al., 1994; Sidanius \& Pratto, 1999). Aunque inicialmente fue conceptualizada como el deseo de que el grupo de uno sea socialmente dominante o relevante (Sidanius et al., 2004), estudios recientes sugieren que no está relacionada con grupos específicos, políticas o contextos, sino que constituye un deseo general de establecer y mantener diferencias entre grupos jerárquicamente estructuradas, independientemente de la posición del propio grupo dentro de esta jerarquía (Ho et al. 2015; Kteily, Ho, \& Sidanius, 2012; Quist \& Resendez, 2010; Pratto et al., 2006).

Las personas con una SDO alta buscan perpetuar la jerarquía social. Un mecanismo mediante el cual los individuos altos en SDO resisten el cambio social igualitario y expresan mayor prejuicio hacia los grupos subordinados, es respaldando ideologías HE (Ho et al., 2015; Ho et al., 2012; Kteily et al., 2012; Sidanius, Cotterill, Sheehy-Skeffington, Kteily \& Carvacho, 2016). Por el otro lado, se encontró que los individuos con una SDO baja respaldan los mitos legitimadores HA. Así, estas personas favorecen la igualdad entre grupos, la inclusión social, demuestran preocuparse más por otras personas y rechazan la segregación (Kteily et al., 2016; Pratto et al., 2006; Pratto et al., 2012).

La alta SDO está relacionada directamente con el racismo, el prejuicio y la persecución de ciertos grupos: aquellos que poseen bajo estatus o poco poder social, minorías étnicas, mujeres y mujeres líderes, extranjeros, inmigrantes y refugiados (Bates \& Heaven, 2001; Castillo \& Montes, 2008; Duckitt, 2006; Jackson \& Esses, 2000; Pratto et al., 1999; Sidanius, Pratto \& Bobo, 1996; Thomsen, Green \& Sidanius, 2008). También predice el apoyo a ciertas ideologías y políticas sociales HE: a políticas y economías conservadoras, la orientación política de 
derecha, las élites culturales, el autoritarismo, la identificación nacionalista, el patriotismo, el sexismo, el mayor apoyo a los militares, la pena de muerte y la tortura, entre otras (Cárdenas, et al., 2009; Duriez \& Van Hiel, 2002; Haley \& Sidanius, 2005; Pratto et al., 1994; Pratto et al., 1997; Sidanius \& Pratto 1999, 2004). Se ha hallado correspondencia entre la SDO y la oposición a políticas sostenibles, a los derechos civiles y al estado benefactor (Sidanius et al., 2004). Además, la SDO se relaciona con la meritocracia o políticas individualistas de progreso (Sibley \& Duckitt, 2010).

\section{Dimensiones de la SDO}

La literatura indica que hay dos aspectos relacionados, pero conceptualmente distintos de la SDO y estos aspectos predicen fenómenos intergrupales cualitativamente distintos (Ho et al., 2012). Aquellos individuos altos en SDO-D, se orientan hacia la dominación manifiesta de algunos grupos hacia otros, y aquellos altos en SDO-E, apoyan de forma más sutil la jerarquía grupal no igualitaria (Ho et al., 2015).

\section{SDO-Dominante}

La dimensión dominante de la SDO representa una preferencia por los grupos basados en jerarquías, en donde los grupos dominantes oprimen activamente a los grupos subordinados. La SDO-D constituye en el apoyo hacia el mantenimiento y opresión activa, incluso violenta, de las jerarquías en las cuales los grupos con mejor estatus dominan y controlan los beneficios de los grupos con menos estatus (Ho et al. 2015; Ho et al., 2012).

En el estudio realizado por Ho y cols. (2015) se encontró que la SDO-D se correlaciona negativamente con la identificación étnica entre los miembros de grupos minoritarios. Los resultados muestran que es poco probable que la SDO-D sea una medida del deseo del dominio del propio grupo, ya que cuanto más se identifica un grupo subordinado, más baja es la SDO. Los autores (Ho et al., 2015) explicitan su desacuerdo con lo planteado por Jost y Thompson (2000), quienes afirman que esta dimensión se basa en el deseo del propio 
grupo de dominar. Por esta razón, en la nueva escala $\mathrm{SDO}_{7}$ (Ho et al., 2015) se quitaron aquellos ítems de la escala que podrían llevar a una confusión entre la SDO-D y la identificación con el propio grupo.

La SDO-D en comparación con la SDO-E predice actitudes intergrupales enérgicas y agresivas dirigidas hacia grupos de menor status: persecución hacia los inmigrantes, apoyo a la guerra, al castigo, a la tortura, a la pena de muerte, y apoyo a la acción militar en Irán e intervención en Siria. También se relaciona directamente con la aprobación de creencias que justifican la opresión, como el racismo clásico, el nacionalismo, el militarismo y la legitimación de la guerra (Bobo \& Hutchings, 1996; Ho et al., 2015).

Kteily, Bruneau, Waytz y Cotterill (2015) encontraron una correlación entre la SDO-D y la deshumanización, conceptualizada como un proceso psicológico que despoja a miembros del exogrupo menos humanamente que a los individuos del propio grupo, pudiendo incluso desencadenar en violencia contra el grupo deshumanizado. Específicamente, los individuos que respaldan la jerarquía (individuos con alta SDO) son más propensos a percibir su grupo como más evolucionado que los grupos externos y negar la humanidad de estos últimos. La deshumanización evidente está significativamente más asociada con SDO-D que cualquiera de las medidas sutiles de deshumanización. Sin embargo, estas últimas están igualmente relacionadas con la SDO-D y la SDO-E (Leyens et al., 2000). Larsson, Björklund y Bäckström (2012) hallaron que la SDO-D tiene un papel clave en la legitimación y el involucramiento hipotético en comportamientos abusivos similares a los de la tortura. Además, la SDO es un posible factor de riesgo comportamiento abusivo, especialmente en situaciones en las que un individuo esté luchando por el poder un grupo, en el que la violencia es un medio aceptable para defender y mantener la superioridad. Asimismo, la SDO-D correlaciona con variables de personalidad específicas como puntajes más bajos en la dimensión de HEXACO en honestidad-humildad (Lee, Ashton, Ogunfowora, Bourdage \& Shin, 2010; Sibley, Harding, Perry, Asbrock \& Duckitt, 2010), "rasgos de la tríada oscura” como maquiavelismo y psicopatía (Hodson, Hogg \& 
MacInnis, 2009) y una visión competitiva del mundo (Duckitt, Wagner, Du Plessis \& Birum, 2002; Perry, Sibley \& Duckitt, 2013). Puntuaciones bajas en la dimensión honestidad-humildad de HEXACO y altas en maquiavelismo, indican que la persona está dispuesta a lograr sus objetivos por medios deshonestos, factores que pueden explicar la sólida relación entre SDO-D y la falta de interés hacia los costos sociales de las medidas utilizadas para conseguir o mantener jerarquías de dominancia grupales.

\section{SDO-Igualitarismo}

La segunda subdimensión de SDO, el anti-igualitarismo, se caracteriza por la oposición sutil a la igualdad entre grupos y el apoyo al acceso diferencial de ciertos grupos al poder y a los recursos. Se manifiesta en una afinidad hacia las ideologías y políticas que mantienen la desigualdad, especialmente hacia aquellas que no involucran confrontación violenta o manifiesta, como la meritocracia (Ho et al., 2015; Ho et al., 2012).

La SDO-E es un mejor predictor de las ideologías y creencias HE que SDO-D. Por ejemplo, del conservadurismo político en Estados Unidos, el apoyo a la distribución desigual de recursos (Ho et al., 2015), la oposición a las políticas HA sobre la ciudadanía y la multiculturalidad (Hindriks et al., 2014) . La SDO-E, en comparación con SDO-D, fue un mejor predictor de la oposición a la discriminación positiva y a políticas dirigidas a la igualdad racial, la oposición al bienestar social y aversión a las profesiones HA (Ho et al., 2015). Por ejemplo, se ha encontrado correlación entre la SDO-E y el afecto negativo hacia los migrantes (Martinovic \& Verkuyten, 2013).

Coherente con la relación entre SDO-E subdimensión y la justificación del sistema, hay una correlación positiva entre SDO-E y conformidad social. Esto implica que, aquellos con alta SDO-E pueden mostrarse de acuerdo con una norma de desigualdad, pero no a una de dominación abierta (Ho et al., 2015).

Las personas que poseen una puntuación alta en la SDO-D y en comparación con aquellas con alta SDO-E tienen preferencia por distintos tipos de desigualdad y procesos para conseguirla y mantenerla. 
Mientras que los individuos altos en SDO-D se inclinan por jerarquías dominantes donde los grupos de alto poder opriman y subyuguen a los grupos inferiores, estando dispuestos a conseguir esta forma de desigualdad mediante el uso de medidas agresivas, los individuos altos en SDO-E anteponen jerarquías en donde los recursos estén injustamente distribuidos, que pueden ser defendidos por ideologías anti-igualitarias. De esta manera, parecería que SDO-D es más severa que SDO-E (Ho et al., 2015). Sin embargo, aunque los conflictos violentos y la opresión llaman la atención, la desigualdad muchas veces es más sostenible cuando es mantenida por formas más sutiles, que abarcan complejas fuentes ideológicas (Jackman, 1994). De acuerdo con Pinker (2011), la coerción para mantener la desigualdad está disminuyendo a nivel global; por lo que las formas más sutiles tomarían su lugar, aumentando el rol de la SDO-E en el futuro. No obstante, el tipo de conflicto intergrupal asociado a SDO-D sigue siendo contemporáneo en las relaciones intergrupales, por lo que esta dimensión de la SDO sigue siendo relevante (Ho et al., 2015).

\section{SDO y variables sociodemográficas SDO y sexo}

La asimetría ideológica implica que las actitudes y preferencias de los grupos dominantes, en comparación con los grupos subordinados, están más dirigidas por los valores de la SDO. Se manifiesta en el género, ya que transculturalmente los hombres obtienen mayores puntuaciones en SDO que las mujeres, asimetría que ha sido denominada como la hipótesis de invarianza de género (Pratto et al., 2006). De esta manera, se encontraría en los hombres un mayor interés por mantener las estructuras jerárquicas establecidas (Pratto et al., 2000; Pratto et al., 1997; Sidanius et al., 2000; Sidanius \& Pratto, 1999; Sidanius et al., 1995).

En los comienzos de la formulación de la hipótesis de la invarianza de género, Sidanius, Pratto y Bobo (1994), afirmaron que, después de considerar factores culturales, situacionales y ambientales, debería haber un promedio más alto de SDO en los hombres que en 
las mujeres. Por lo tanto, se afirmó que los niveles de SDO eran muy estables y poco dependientes del entorno, ya que se basan en estrategias reproductivas desiguales asumidas por hombres y por mujeres. Estos postulados son objeto de debate en la actualidad. Las principales objeciones giran en torno a la relevancia otorgada a los componentes biológicos y la ausencia de integración con otras variables socioculturales. Se identificaron otras condiciones que moderan la diferencia de género en la SDO, como la identificación de género, la autoatribución de rasgos estereotípicos masculinos y femeninos, y la socialización del género. A partir de estas críticas, la SDT adoptó una visión menos determinista según la cual las diferencias entre género y en el nivel de SDO son el resultado de la interacción compleja entre genotipos y entornos culturales y sociales, y que a su vez puede manifestarse en los roles de género (Aranda, Montes-Berges \& Castillo-Mayén, 2015).

En un estudio realizado por Lee y cols. (2011) en donde se evaluaron los moderadores de las diferencias de género en la SDO en 22 países, en ninguna cultura las mujeres tuvieron puntuaciones significativamente más altas en SDO que los hombres. Además, se halló que en las sociedades que poseen más igualdad de género, las mujeres rechazan la jerarquía basada en grupos más que los hombres. Este hallazgo sugiere que las mujeres que asumen que pueden ser iguales a los hombres están más sensibilizadas a la desigualdad social. Además, puede ser que cuando las mujeres acceden a profesiones que clásicamente ocuparon los hombres, su experiencia confirma lo desigual que son tratadas ellas y otros grupos. Otra razón para la correlación entre la igualdad de género en las sociedades y las diferencias de género en la SDO puede ser que ambas fueron provocadas por el activismo feminista, donde las mujeres (presumiblemente en especial las que tienen bajos niveles de SDO) han luchado por la igualdad, creando oportunidades para la igualdad de la educación superior, igualdad salarial y roles profesionales para las mujeres, así como para difundir y vivir de acuerdo a una ideología anti-dominante. Tal trabajo político puede cambiar tanto la SDO, como el grado de desigualdad en una sociedad. 
En Argentina, se encontraron puntuaciones significativamente más altas de SDO en hombres que en mujeres (Achaval, 2011; Muratori, et al., 2012; Petit \& Costa, 2011).

\section{SDO y edad}

No existen numerosos estudios previos que exploren la relación entre edad y SDO. Sin embargo, se espera que a mayor juventud predominen ideas igualitarias por sobre ideas de dominación social (Zubieta et al., 2007). De acuerdo con las formulaciones teóricas de la SDT, las jerarquías basadas en la edad existen invariablemente en todos los sistemas sociales, donde las personas de edad media tienden a poseer un poder desproporcionado con respecto a las más jóvenes (Pratto et al., 2006). En Argentina, no se han encontrado relaciones significativas entre edad y SDO (Zubieta et al., 2007).

\section{SDO y autoposicionamiento socioeconómico}

Debido a que aquellas personas pertenecientes a los grupos dominantes se benefician más de la jerarquía grupal, se expecta que los miembros de estos tiendan a respaldar más la dominancia social, es decir, a poseer niveles más altos de SDO en comparación con aquellos de los grupos subordinados. Esta hipótesis se denomina asimetría ideológica (Pratto et al., 2006; Sidanius et al., 1994).

\section{SDO y orientación política}

La SDO está relacionada con el respaldo de las ideologías sociales del conservadurismo político-económico, del militarismo y el nacionalismo, es decir, creencias HE (Ho et al., 2015; Pratto et al., 1994). Además, SDO se correlaciona positivamente con las creencias que apoyan la jerarquía basada en grupos (como, por ejemplo, la meritocracia y el racismo; Pratto et al., 1994). Esto también se observó en el contexto argentino (Zubieta et al., 2007).

El objetivo general de este trabajo fue adaptar la séptima versión de la Escala de Orientación a la Dominancia Social en su forma larga 
(16 ítems) y corta, (ocho ítems). Nuestros objetivos específicos fueron los siguientes. Primero, analizar la consistencia interna de la escala $\mathrm{SDO}_{7}$ en sus dos versiones y evaluar su estructura factorial, esperando encontrar una estructura bifactorial de la $\mathrm{SDO}_{7}$. Segundo, comparar los niveles de SDO en función de las variables de sexo, edad, autoposicionamiento socioeconómico y orientación política. Se predice que los niveles de SDO en los hombres son más altos que en las mujeres; que a mayor edad más altas son las puntuaciones en SDO; que las personas que se perciben perteneciendo a un nivel socioeconómico más alto presenten puntuaciones más elevadas en SDO que las que se perciben perteneciendo a un nivel socioeconómico más bajo; y que, las personas con orientación política de derecha exhiben puntuaciones más altas en SDO que los de izquierda. Estas hipótesis aplican para las dos versiones y subdimensiones.

\section{Método}

Se trata de un estudio descriptivo correlacional, de diseño no experimental transversal, con sujetos residentes en la zona metropolitana de Buenos Aires de ambos sexos entre 18 y 60 ańos como unidad de análisis.

\section{Participantes}

Se realizó un muestreo no probabilístico intencional compuesto por 1573 participantes voluntarios (57.6\% mujeres y $42.4 \%$ hombres), residentes de la zona metropolitana de Buenos Aires. Todos contestaron la escala $\mathrm{SDO}_{7}$ versión corta (ocho ítems) al tiempo que, de estos, 590 completaron los ocho ítems restantes que componen la escala larga de 16 ítems. Sus edades fluctúan entre 18 y 60 años $(M=28.08 ; D E$ = 12.14). Respecto de su nivel socioeconómico autopercibido, siendo 1 "muy pobre" y 7 "muy rico", la media fue de $4.32(D E=1.14)$. Finalmente, su orientación política, considerando que 1 es "extrema 
izquierda" y 7 "extrema derecha", tendió más hacia la derecha $(\mathrm{M}=$ 4.57; $D E=1.55)$.

\section{Medición}

Para recolectar los datos se utilizó como instrumento la Escala $\mathrm{SDO}_{7}$ (Ho et al., 2015) adaptada y traducida al espańol por medio de la traducción inversa, siguiendo las pautas internacionales metodológicas para la traducción y adaptación de tests, planteadas por la International Test Comission (2005). Un traductor realizó una traducción de la escala desde el inglés al español y, luego, un segundo lingüista tomó el test traducido en español y se volvió a traducir al idioma original. Posteriormente, se compararon ambas versiones, llegándose a una versión final por consenso.

El formato de la $\mathrm{SDO}_{7}$ corresponde a una escala tipo Likert con siete alternativas de respuesta, presentadas en un rango de 1 = "me opongo fuertemente" a 7 = "estoy fuertemente a favor". Cuenta con 16 ítems como, por ejemplo: "Una sociedad ideal requiere que algunos grupos estén por encima y que otros estén por debajo", para medir la SDO-D, y "Es injusto intentar hacer iguales a los grupos", para medir la SDO-E. La escala corta, compuesta por ocho ítems presentes en la versión larga, utiliza la misma metodología. Una vez invertidos los ítems, los valores más altos indican una mayor tendencia a la dominancia social. De esta forma, ambas versiones tienen dos dimensiones y al interior de estas se incluyen igual cantidad de ítems directos e inversos.

La puntuación para el valor de la SDO total se obtiene revirtiendo los ítems 5, 6, 7, 8, 13, 14, 15 y 16, sumando las puntuaciones de todas las respuestas dadas y dividiendo el resultado por 16. En el caso de la versión corta, el valor total se obtiene revirtiendo los ítems 3, 4, 7 y 8, y aplicando el mismo procedimiento que para la versión extendida, en este caso dividiendo por 8 . El valor de cada subdimensión se consigue invirtiendo los ítems correspondientes, en el caso de la SDO-D para la versión reducida los ítems 3 y 4 y para la versión extendida los ítems 5 , 6, 7 y 8; y para la SDO-E en la primera versión se invierten los ítems 7 y 8; y en la larga, los ítems 13, 14, 15 y 16, luego se suman todas las 
respuestas y se dividen por 4 o por 8 , según corresponda. El detalle de los ítems puede verse en la Tabla 1.

\section{Tabla 1}

Ítems de la escala $\mathrm{SDO}_{7}$ en su versión larga y corta

\begin{tabular}{|c|c|c|}
\hline \multicolumn{2}{|c|}{ Numeración } & \multirow[t]{2}{*}{ Ítems } \\
\hline Larga & Corta & \\
\hline 1 & -- & Algunos grupos de personas deben mantenerse en su lugar. \\
\hline 2 & -- & $\begin{array}{l}\text { Probablemente sea bueno que ciertos grupos estén en la parte } \\
\text { superior y otros grupos estén en la parte inferior. }\end{array}$ \\
\hline 3 & 1 & $\begin{array}{l}\text { Una sociedad ideal requiere que algunos grupos estén por } \\
\text { encima y que otros estén por debajo. }\end{array}$ \\
\hline 4 & 2 & $\begin{array}{l}\text { Algunos grupos de personas son simplemente inferiores a } \\
\text { otros grupos. }\end{array}$ \\
\hline $5 \mathrm{R}$ & $3 \mathrm{R}$ & $\begin{array}{l}\text { Los grupos que están en la parte inferior de la sociedad } \\
\text { son tan merecedores como los grupos que están en la parte } \\
\text { superior. }\end{array}$ \\
\hline $6 \mathrm{R}$ & $4 \mathrm{R}$ & Ningún grupo debe dominar en la sociedad. \\
\hline $7 \mathrm{R}$ & -- & $\begin{array}{l}\text { Los grupos que están en la parte inferior de la sociedad no } \\
\text { deberían permanecer en su lugar. }\end{array}$ \\
\hline $8 \mathrm{R}$ & -- & La dominancia grupal es una idea poco elaborada, mediocre. \\
\hline 9 & -- & No debemos presionar para tener igualdad grupal. \\
\hline 10 & -- & $\begin{array}{l}\text { No debemos tratar de garantizar que cada grupo tenga la } \\
\text { misma calidad de vida. }\end{array}$ \\
\hline 11 & 5 & Es injusto intentar hacer iguales a los grupos. \\
\hline 12 & 6 & La igualdad grupal no debe ser nuestro objetivo principal. \\
\hline $13 \mathrm{R}$ & $7 \mathrm{R}$ & $\begin{array}{l}\text { Debemos trabajar para dar a todos los grupos de las mismas } \\
\text { oportunidades de éxito. }\end{array}$ \\
\hline $14 \mathrm{R}$ & $8 \mathrm{R}$ & $\begin{array}{l}\text { Debemos hacer lo que podamos para igualar las condiciones } \\
\text { de los diferentes grupos. }\end{array}$ \\
\hline
\end{tabular}


No importa la cantidad de esfuerzo que se requiera, tenemos

$15 \mathrm{R} \quad---\quad$ que tratar de asegurar que todos los grupos tengan las mismas oportunidades en la vida.

16R --- $\quad$ La igualdad grupal debe ser nuestro ideal.

Nota. Los ítems que presentan una $\mathrm{R}$ al lado el número son los ítems que deben ser invertidos para calcular el valor de la escala total y de sus subescalas.

Las investigaciones realizadas por Ho y cols. (2015) en población estadounidense evidencian una adecuada estructura factorial para las dos versiones y niveles satisfactorios de consistencia interna con valores entre $\alpha=.89$ y $\alpha=.95$ en la más extensa y entre $\alpha=.78$ y $\alpha=.90$ en la versión más reducida.

Se incluyó un cuestionario para evaluar datos sociodemográficos en donde se consignaron preguntas para obtener información sobre el sexo, la edad, nivel socioeconómico autopercibido y orientación política.

\section{Procedimiento}

Para llegar a una muestra amplia y heterogénea, se recolectaron los datos a través de Internet, enviando un e-mail a conocidos, que a su vez contactaron a otros conocidos (método "bola de nieve"), donde se proporcionó un link desde el cual se accedía al cuestionario online. La escala autoadministrada se completó de forma individual y el tiempo promedio necesario para completarla fue de 15 minutos aproximadamente. La participación fue voluntaria y anónima, y las respuestas fueron recabadas durante el lapso de 25 días. Antes de comenzar con la encuesta, los participantes debían firmar un consentimiento informado, que cumple con los lineamientos para el comportamiento ético en las Ciencias Sociales y Humanas establecidos por el Consejo Nacional de Investigaciones Científicas y Técnicas (CONICET) de Argentina (Res. D N 2857/06), en el cual se les informó acerca de los propósitos de la investigación. 


\section{Análisis de datos}

Los datos obtenidos fueron codificados y analizados a través del programa Statistical Package for the Social Sciences - SPSS versión 25. Para conocer la consistencia interna de las dos versiones de la escala, se calculó el alfa de Cronbach. Para obtener evidencias acerca de la estructura bifactorial de la escala, se efectuó un análisis factorial confirmatorio a través del paquete AMOS versión 20. Para conocer la relación entre SDO y edad, nivel socioeconómico autopercibido y orientación política se utilizó el estadístico $r$ de Pearson y para comparar a los participantes según el sexo se aplicó la prueba $t$ de Student, calculándose en este último caso el tamaño del efecto utilizando el programa G*Power 3.1.9.4. Se utilizaron estos estadísticos paramétricos ya que, según la Teoría Central del Límite, se asume una distribución normal cuando el n es grande (Szretter-Noste, 2013).

\section{Resultados}

\section{Confiabilidad}

La tabla 2 presenta el coeficiente $\alpha$ de Cronbach y la media y desvío estándar para la escala total y las dos dimensiones de las versiones larga y corta. Los participantes presentan niveles bajos de SDO. $\mathrm{Al}$ analizar la consistencia interna de la versión argentina de la $\mathrm{SDO}_{7}$, se halló una fiabilidad de $\alpha$ de Cronbach $=.83$ en la escala de 16 ítems, y de $\alpha=.70$ en la escala corta. Estos resultados son coherentes con la observación de Ho y cols. (2015) donde la escala completa tiene mejor consistencia interna que en su versión corta. Al comparar los valores de los $\alpha$ obtenidos aquí con las seis muestras presentadas por Ho y cols. (2015) se observa que en general la fiabilidad obtenida es menor en la presente muestra. Sin embargo, se conservan los niveles aceptables mínimos de fiabilidad para la versión larga, siendo la fiabilidad de la escala corta más baja. 


\section{Tabla 2}

Confiabilidad y niveles de $\mathrm{SDO}_{7}$ para la escala larga y corta en sus dos dimensiones y diferencia según sexo

\begin{tabular}{|c|c|c|c|c|c|c|}
\hline & $\alpha$ & $M$ & $D E$ & Hombre & Mujer & $\begin{array}{l}\text { Estadístico de diferencias de } \\
\text { media y tamańo efecto }\end{array}$ \\
\hline $\mathrm{SDO}_{7}$ total & .83 & 2.92 & 0.99 & 3.05 & 2.80 & $\begin{array}{l}t(588)=-3.15 ; p=.002 ; \\
\text { IC95\% }[-0.41 ;-0.95] ; d=0.25 .\end{array}$ \\
\hline $\mathrm{SDO}_{7}$ corta & .70 & 2.92 & 1 & 3.07 & 2.80 & $\begin{array}{l}t(1338.85)=-5.08 ; p<.000 ; \\
\text { C95\% }[-0.36 ;-0.16] ; d=0.27 .\end{array}$ \\
\hline $\mathrm{SDO}_{7}-\mathrm{D}$ total & .68 & 3.15 & 1.06 & 3.23 & 3.06 & $\begin{array}{l}t(573.34)=-1.98 ; p=.047 \\
\text { IC95\% }[-0.34 ;-0.01] ; d=0.16 .\end{array}$ \\
\hline $\mathrm{SDO}_{7}-\mathrm{D}$ corta & .50 & 3.09 & 1.20 & 3.22 & 2.98 & $\begin{array}{l}t(1571)=-3.79 ; p<.000 \\
\text { IC95\% }[-0.35 ;-0.11] ; d=0.20\end{array}$ \\
\hline $\mathrm{SDO}_{7}-\mathrm{E}$ total & .80 & 2.70 & 1.16 & 2.87 & 2.53 & $\begin{array}{l}t(588)=-3.53 ; p<.000 ; \\
\text { IC95\% }[-0.52 ;-0.14] ; d=0.32 .\end{array}$ \\
\hline $\mathrm{SDO}_{7}-\mathrm{E}$ corta & .64 & 2.75 & 1.15 & 2.92 & 2.62 & $\begin{array}{l}t(1354.37)=-5.03 ; p<.000 ; \\
\text { IC95\% }[-0.41 ;-0.18] ; d=0.30\end{array}$ \\
\hline
\end{tabular}

\section{Análisis factorial confirmatorio (AFC)}

Con el objetivo de analizar las dos subdimensiones (SDO-D y SDO-E) en las dos versiones de la escala, se efectuó un AFC que permite una fuerte aproximación a la definición y/o validación de un constructo (Pérez-Gil, Chacón Moscoso \& Moreno Rodríguez; 2000). Siguiendo lo propuesto por Ho et al. (2015), los modelos hipotetizados, consideran las dimensiones sustantivas (SDO-D y SDO-E) y de "método" (ítems directos e inversos). Estos se pueden observar en las Figuras 1 (versión larga) y 2 (versión corta).

La Tabla 3 presenta los índices de ajuste y error para cada versión de la escala $\mathrm{SDO}_{7}$. Las dos versiones muestran un nivel de ajuste muy bueno, y acorde a la hipótesis planteada relativa a que la $\mathrm{SDO}_{7}$ en sus dos versiones posee una estructura bifactorial. 


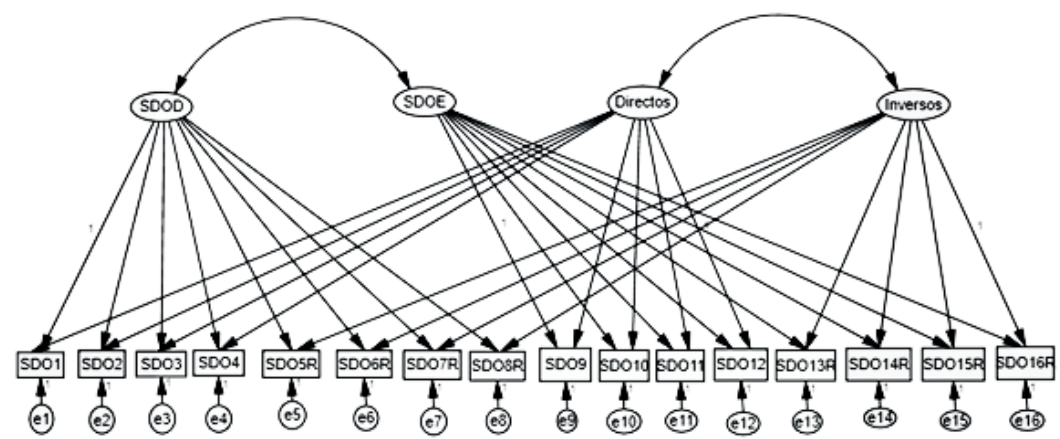

Figura 1. Modelo hipotetizado para la versión larga de $\mathrm{SDO}_{7}$

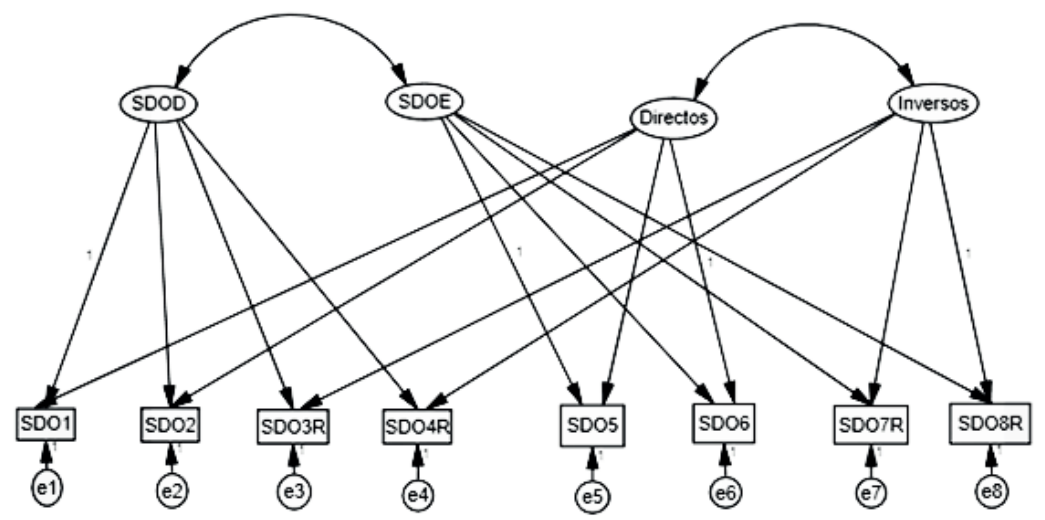

Figura 2. Modelo hipotetizado para la versión corta de $\mathrm{SDO}_{7}$

\section{Tabla 3}

Indices de ajuste y error según AFC para las versiones corta y larga

\begin{tabular}{ccccc}
\hline Versión & $\mathrm{N}$ & CFI & RMSEA & Chi cuadrado \\
\hline $\mathrm{SDO}_{7}$ larga & 590 & .965 & .041 & $\chi^{2}(86)=171.811 ; p<.001 . \chi^{2} / \mathrm{gl}=1.9978$ \\
$\mathrm{SDO}_{7}$ corta & 1573 & .988 & .040 & $\chi^{2}(10)=34.549 ; p<.001 . \chi^{2} / \mathrm{gl}=3.4549$ \\
\hline
\end{tabular}




\section{Diferencias según variables sociodemográficas}

De acuerdo a nuestra hipótesis, se encontraron diferencias estadísticamente significativas ${ }^{4}$ entre los niveles de SDO en hombres y mujeres, tanto en las dos versiones de la escala como en sus subdimensiones (ver Tabla 1 ).

La tabla 4 presenta los coeficientes de correlación entre la SDO en sus distintas versiones y subdimensiones, la edad, el autoposicionamiento socioeconómico y la orientación política de los participantes. Refutando nuestra hipótesis, no se halló relación estadísticamente significativa en lo que respecta al nivel de SDO de los participantes y su edad o autoposicionamiento socioeconómico en ninguna de las versiones de la escala y subdimensiones. Acorde a nuestras predicciones, se halló correlación positiva entre el autoposicionamiento político de derecha y la SDO tanto para las versiones larga y corta y las dos subdimensiones.

\section{Tabla 4}

Correlaciones entre SDO, edad, nivel socioeconómico y orientación politica

\begin{tabular}{lcccccc}
\hline & \multicolumn{2}{c}{ Edad } & \multicolumn{3}{c}{ Nivel socioeconómico } & \multicolumn{3}{c}{ Orientación política } \\
& $r$ & $p$ & $r$ & $p$ & $r$ & $\mathrm{p}$ \\
\hline SDO $_{7}$ total & -.011 & .790 & .046 & .261 & .297 & $<.001$ \\
SDO $_{7}$ corta & -.042 & .095 & .077 & .002 & .288 & $<.001$ \\
SDO-D total & -.026 & .522 & .015 & .716 & .254 & $<.001$ \\
SDO-D corta & -.054 & .033 & .084 & .001 & .265 & $<.001$ \\
SDO-E total & .006 & .893 & .065 & .114 & .273 & $<.001$ \\
SDO-E corta & -.018 & .484 & .048 & .059 & .228 & $<.001$ \\
\hline
\end{tabular}

Nota . Para la versión larga $\mathrm{N}=590$ y para la versión corta $\mathrm{N}=1573$. Teniendo en cuenta el gran tamaño de la muestra para la versión corta, se considera una significación de $p<, 001$

4 Teniendo en cuenta el gran tamańo de la muestra para la versión corta $(n=1573)$, se considera una significación de $p<.001$. 


\section{Discusión}

El principal objetivo del presente trabajo fue la adaptación al castellano de la séptima escala de la dominancia social en Argentina (Ho et al., 2015), el estudio de su consistencia interna, validez factorial e incidencia con respecto a las variables de sexo, edad, autoposicionamiento socioeconómico y orientación política.

En este estudio se han hallado niveles bajos de SDO, semejantes a los encontrados en investigaciones previas argentinas (Achaval, 2011; Beramendi \& Zubieta, 2016; Muratori et al., 2012; Petit \& Costa, 2011; Zubieta, Beramendi, Sosa \& Torres., 2011; Zubieta et al., 2007). Una posible explicación de este fenómeno es que la SDO tiende a ser más baja en los países más democráticos e igualitarios (Fischer, Hanke \& Sibley, 2012).

En consonancia con los estudios nacionales e internacionales (Cárdenas et al., 2010; Ho et al., 2015) Jost \& Thompson, 2000; Silván-Ferrero \& Bustillos, 2007) y nuestra primera hipótesis, estructura factorial de la escala tiene un ajuste bidimensional. Por lo tanto, el constructo SDO posee dos dimensiones: la SDO dominante y la SDO anti-igualitaria. En futuras investigaciones sería interesante comprobar la importancia de cada uno de los factores en el mantenimiento de las actitudes hacia diferentes grupos. Respecto a la consistencia interna de la escala, se observa un alfa de Cronbach de ,83 para la escala en su versión larga $(n=590)$. Este valor es menor al de la versión original del instrumento, que obtuvo valores entre $\alpha=.89$ y $\alpha=.95$ (Ho et al., 2015). Sin embargo, es similar a las adaptaciones anteriores de la $\mathrm{SDO}_{6}$ en países de habla hispana, en Espańa ( $\alpha=.85$; Silván-Ferrero y Bustillos, 2007), en Chile ( $\alpha=.86$; Cárdenas et al., 2010) y en Argentina $(\alpha=.85$; Etchehazar et al., 2014). Para la escala corta, Ho y cols. (2015) encontraron menores niveles de fiabilidad en comparación con la versión larga (entre $\alpha=.78$ y $\alpha=.90$ ), al igual que los que se hallaron en este estudio $(\alpha=.70)$. Por lo tanto, $\mathrm{SDO}_{7}$ goza de una apropiada consistencia interna $y$ es un instrumento fiable para medir la SDO en nuestro contexto. 
Respecto a las dos subdimensiones, en la versión original del instrumento (Ho et al., 2017) los valores de confiabilidad se hallaron dentro de los parámetros esperables. Sin embargo, en cinco de las seis muestras que se realizaron, los valores fueron mayores para la SDO-E en comparación de la SDO-D. Estos resultados coinciden con los obtenidos en el presente estudio donde $\alpha=.68$ para SDO-D y $\alpha=.80$ para SDO-E en la versión de 16 ítems.

De acuerdo con nuestra hipótesis y la hipótesis de la invarianza de género, los hombres puntuaron más alto en SDO que las mujeres. Estos resultados coinciden con estudios anteriores en Argentina, donde se encontraron mayores niveles de SDO en los hombres que en las mujeres (Achaval, 2011; Muratori, et al., 2012; Petit \& Costa, 2011). Este hallazgo implica que las actitudes y preferencias del grupo dominante (que en este caso sería el grupo de los hombres), están más fuertemente dirigidas por valores de dominancia social que en los miembros del grupo subordinado, las mujeres. Además, los hombres obtuvieron niveles más elevados en la SDO-D y SDO-E, que implica que sus actitudes hacia la desigualdad, son más influenciadas por mitos HE que sustentan el sistema de desigualdad de sexos entre grupos y el rechazo de aquellas políticas que favorezcan la igualdad grupal (SilvánFerrero \& Bustillos, 2015).En estudios posteriores sobre esta temática, será relevante investigar cómo esta asimetría se ve influenciada por el movimiento feminista, ya que desde el 2015 la potencia colectiva de este movimiento de mujeres magnificó su importancia en el país (Pozzo, 2018). Esta ideología es claramente un desafío para las jerarquías grupales debido a que el feminismo es una ideología política que apoya la igualdad de trato de todas las personas independientemente de los juicios categóricos (Unger, 1979). Por lo tanto, se esperaría que las creencias feministas estén inversamente relacionadas con SDO (Foels \& Pappas, 2004).

En lo que refiere a la edad, los resultados no corroboran la hipótesis planteada. En otro estudio argentino (Zubieta et al., 2007), tampoco se encontró relación entre la edad y la SDO. En lo que hace a la relación entre SDO y nivel socioeconómico la hipótesis planteada también 
es rechazada, no se verifica relación entre las variables. La correlación positiva entre SDO y orientación política de derecha va en línea con la hipótesis de asimetría ideológica: las ideologías y actitudes que mejoran la jerarquía son impulsadas más fuertemente por los grupos dominantes. Así, los sujetos de derecha manifiestan más actitudes que tienen como objetivo mantener y perpetuar la desigualdad (Cárdenas et al, 2010).

El presente estudio no está exento de limitaciones. En primer lugar, la muestra utilizada fue de tipo no probabilístico intencional. Por otra parte, en lo que hace a la distribución de la muestra, si bien se trabajó con una alta cantidad de participantes $(n=1573)$, el $55 \%$ de esta son jóvenes de que se encuentran entre los 18 y los 22 años. Otra de las limitaciones de esta investigación, es que no se ha estudiado la validez de criterio de la escala, que en estudios anteriores se ha evaluado mediante sus relaciones con el autoritarismo del ala de derechas (RWA; Cárdenas et al., 2010; Etchezahar et al., 2014; Silván-Ferrero \& Bustillos, 2007). Este se define como la covariación de tres conglomerados actitudinales: sumisión autoritaria, agresión autoritaria y convencionalismo (Altemeyer, 1998; Duckitt, 2006). La necesidad de estudiar ambos constructos de manera conjunta se debe a que cada uno de ellos remite a una dinámica específica de las relaciones intergrupales (Duckitt, 2006). Mientras que el RWA se focaliza en el grado de adhesión endogrupal (por ejemplo, normas internas, líderes), la SDO especifica la diferenciación con exogrupos (Duckitt, 2006; Zakrisson, 2005).

$\mathrm{La}$ adaptación de la escala $\mathrm{SDO}_{7}$ para la Argentina marca un antecedente para el estudio y la comprensión de la desigualdad y del prejuicio en la sociedad. Además, es útil en los casos en que una ideología o política relevante del grupo es ambigua, ya que utilizando la escala completa y luego examinando la relación entre orientación ideológica o política y cada subdimensión de la SDO, puede ayudar a proporcionar claridad sobre las motivaciones subyacentes. Por lo tanto, el uso de la escala $\mathrm{SDO}_{7}$ puede ayudar a los investigadores a interpretar de forma precisa la desigualdad que una orientación política o ideología en particular pretende sustentar. Asimismo, $\mathrm{SDO}_{7}$ posee una 
validez predictiva diferencial que permite a los investigadores estudiar únicamente el aspecto de la SDO (Dominancia o Igualitarismo) que más se corresponda con los fenómenos intergrupales en los que están interesados. Por ejemplo, quienes estén enfocados en conflictos caracterizados por un alto grado de opresión o actitudes extremas, pueden recurrir a utilización de la SDO-D, mientras que los investigadores interesados en políticas sociales relacionadas con la redistribución de recursos pueden focalizarse en la SDO-E. De esta manera, $\mathrm{SDO}_{7}$ brinda mayor precisión a las investigaciones que involucran dinámicas específicas de jerarquía intergrupal (Ho et al., 2015). Es relevante también la introducción $\mathrm{SDO}_{7}$ en su versión corta, que posee propiedades psicométricas similares a las de la formulación original, ya que esta versión abreviada es una herramienta útil para el estudio de la SDO cuando es posible administrar la escala completa.

\section{Referencias}

Achaval, L. (2011). Orientación a la dominancia social en estudiantes universitarios (Tesis de Licenciatura). Pontificia Universidad Católica Argentina, Buenos Aires.

Aiello, A., Passini, S., Tesi, A., Morselli, D. \& Pratto, F. (2017). Measuring support for intergroup hierarchies: Assessing the psychometric proprieties of the Italian Social Dominance Orientation Scale. $_{7}$ Testing, Psychometrics, Methodology in Applied Psychology, 26(3), 373-383. https://doi.org/10.4473/TPM.26.3.4

Altemeyer, B. (1998). The authoritarian specter. Cambridge, MA: Harvard University Press.

Aranda, M. B., Montes-Berges, B. \& Castillo-Mayén M. R. (2015). La orientación a la dominancia social en la adolescencia: el papel de la identidad de género y los rasgos estereotípicos femeninos y masculinos. Revista de Psicología Social, 30(2), 251-263. https:// doi.org/10.1080/21711976.2015.1016752 
Arifianto, M. H. T. (2018). Orientasi dominasi sosial sebagai alternatif untuk melihat sikap implisit terhadap sistem sosial yang timpang: Adaptasi skala orientasi dominasi sosial7 ( $\mathrm{SDO}_{7}$ scale). Jurnal Psikologi Sosial, 15(2), 105-121. https://doi.org/10.7454/ jps.2017.10

Bates, C. \& Heaven, P. (2001). Attitudes to women in society: the role of social dominance orientation and social values. Journal of Community \& Applied Social Psychology, 11, 43-49. https://doi. org/10.1002/casp.589

Beramendi, M. \& Zubieta, E. (2016). Una nueva perspectiva sobre las creencias igualitarias: contra-dominancia social. Ciencias Psicológicas, 10(1), 43-53. https://doi.org/10.22235/cp.v10i2.1152

Bishop, G. F., Tuchfarber, A. J. \& Oldendick, R. W. (1978). Change in the structure of American political attitudes: The nagging question of question wording. American Journal of Political Science, 22, 250-269. https://doi.org/10.2307/2110616

Bobo, L. \& Hutchings, V. L. (1996). Perceptions of racial group competition: Extending Blumer's theory of group position to a multiracial social context. American Sociological Review, 61, 951-972. https://doi.org/10.2307/2096302

Cárdenas, M., Meza, P., Lagues, K. \& Yañez, S. (2009). Adaptación y validación de la Escala de Orientación a la Dominancia Social (SDO) en una muestra chilena. Universitas Psychologica, 9, 161-168. https://doi.org/10.11144/Javeriana.upsy9-1.aveo

Castillo Mayén, M. \& Montes Berges, B. (2008). Aportaciones de la teoría de la dominancia social al análisis de la discriminación de género. Iniciación a la Investigación, 3. Recuperado de https:// revistaselectronicas.ujaen.es/index.php/ininv/article/view/200 el día 11 de febrero del 2020.

Del Prado, M. \& Bustillos, A. (2007). Adaptación de la Escala de Orientación a la Dominancia Social al castellano: validación de la Dominancia Grupal y la Oposición a la igualdad como factores subyacentes. Revista Psicología Social, 22(1), 3-16. https:// doi.org/10.1174/021347407779697485 
Duckitt, J. (2006). Differential effects of right wing authoritarianism and social dominance orientation on outgroup attitudes and their mediation by threat from and competitiveness to outgroups. Personality and Social Psychology Bulletin, 32, 684-696. https:// doi.org/10.1177/0146167205284282

Duckitt, J., Wagner, C., Du Plessis, I. \& Birum, I. (2002). The psychological bases of ideology and prejudice: Testing a dual process model. Journal of Personality and Social Psychology, 83, 75-93. https://doi.org/10.1037/0022-3514.83.1.75

Duriez, B. \& Van Hiel, A. (2002). The march of modern fascism: A comparison of social dominance orientation and authoritarianism. Personality and Individual Differences, 32, 1199-1213. https://doi.org/10.1016/S0191-8869(01)00086-1

Etchezahar, E., Prado-Gascó, V., Jaume, L. \& Brussino, S. (2014). Validación argentina de la Escala de Orientación a la Dominancia Social. Revista Latinoamericana de Psicología, 46(1), 35-43. https://doi.org/10.1016/S0120-0534(14)70004-4

Fischer, R., Hanke, K. \& Sibley, C. (2012). Cultural and Institutional Determinants of Social Dominance Orientation Across 27 Societies. Political Psychology, 33(4), 437-467. https://doi. org/10.1111/j.1467-9221.2012.00884.x

Fiske, A. P. (1992). The four elementary forms of sociality: Framework for a unified theory of social relations. Psychological Review, 99(4), 689-723. https://doi.org/10.1037/0033-295X.99.4.689

Foels, R. \& Pappas, C. J. (2004). Learning and unlearning the myths we are taught: Gender and social dominance orientation. Sex roles, 50(11-12), 743-757. https://doi.org/10.1023/ B:SERS.0000029094.25107.d6

Haley, H. \& Sidanius, J. (2005). Person-organization congruence and the maintenance of group-based social hierarchy: A social dominance perspective. Group Processes \& Intergroup Relations, 8, 187-203. https://doi.org/10.1177/1368430205051067

Hindriks, P., Verkuyten, M. \& Coenders, M. (2014). Dimensions of social dominance orientation: The roles of legitimizing myths 
and national identification. European Journal of Personality 28(6), 538-549. https://doi.org/10.1002/per.1955

Ho, A. K., Sidanius, J., Pratto, F., Levin, S., Thomsen, L., Kteily, N. \& Sheehy-Skeffington, J. (2012). Social dominance orientation: Revisiting the structure and function of a variable predicting social and political attitudes. Personality and Social Psychology Bulletin, 38, 583-606. https://doi.org/10.1177/0146167211432765

Ho, A. K., Sidanius, J., Kteily, N., Sheehy-Skeffington, J., Pratto, F., Henkel, K. E., Foels, R. \& Stewart, A. L. (2015). The nature of social dominance orientation: Theorizing and measuring preferences for intergroup inequality using the new $\mathrm{SDO}_{7}$ scale. Journal of Personality and Social Psychology, 109(6), 1003-1028. https://doi.org/10.1037/pspi0000033

Hodson, G., Hogg, S. M. \& MacInnis, C. C. (2009). The role of "dark personalities" (narcissism, Machiavellianism, psychopathy), Big Five personality factors, and ideology in explaining prejudice. Journal of Research in Personality, 43, 686-690. https://doi. org/10.1016/j.jrp.2009.02.005

International Test Commission (2005). International Guidelines on Test Adaptation. Recuperado de www.intestcom.org el día 06 de mayo del 2017.

Jackman, M. R. (1994). The velvet glove: Paternalism and conflict in gender, class, and race relations. Oakland, CA: University of California Press.

Jackson, L. M. \& Esses, V. M. (2000). Effects of perceived economic competition of people's willingness to help empower immigrants. Group Processes and Intergroup Relations, 3(4), 419-435. https:// doi.org/10.1177/1368430200003004006

Jost, J. \& Banaji, M. R. (1994). The role of stereotyping in systemjustification and the production of false consciousness. British Journal of Social Psychology, 33, 1-27. https://doi. org/10.1111/j.2044-8309.1994.tb01008.x

Jost, J. \& Thompson, E. (2000). Group-based dominance and opposition to equality as independent predictors of self-esteem, 
ethnocentrism, and social policy attitudes among African Americans and European Americans. Journal of Experimental Social Psychology, 36, 209-232. https://doi.org/10.1006/ jesp.1999.1403

Kteily, N., Bruneau, E., Waytz, A. \& Cotterill, S. (2015). The ascent of man: Theoretical and empirical case for blatant dehumanization. Journal of Personality and Social Psychology, 109(5), 901-31. https://doi.org/10.1037/pspp0000048

Kteily, N., Ho, A. K. \& Sidanius, J. (2012). Hierarchy in the mind: The predictive power of social dominance orientation across social contexts and domains. Journal of Experimental Social Psychology, 48, 543-549. https://doi.org/10.1016/j.jesp.2011.11.007

Kteily, N., Sheehy-Skeffington, J. \& Ho, A. K. (2016) Hierarchy in the eye of the beholder: (anti-) egalitarianism shapes perceived levels of social inequality. Journal of Personality and Social Psychology,112(1), 136-159. http://doi.org/10.1037/ pspp0000097

Larsson, M. R., Björklund, F. \& Bäckström, M. (2012). Rightwing authoritarianism is a risk factor of torture-like abuse, but so is social dominance orientation. Personality and Individual Differences, 53, 927-929. http://doi.org/10.1016/j. paid.2012.06.015

Lee, I., Pratto, F. \& Johnson, B. (2011). Intergroup Consensus/ Disagreement in Support of Group-Based Hierarchy: An Examination of Socio-Structural and Psycho-Cultural Factors. Psychological Bulletin, 137(6), 1029-1064. http://doi. org/10.1037/a0025410

Lee, K., Ashton, M. C., Ogunfowora, B., Bourdage, J. S. \& Shin, K. (2010). The personality bases of socio-political attitudes: The role of honesty-humility and openness to experience. Journal of Research in Personality, 44, 115-119. http://doi. org/10.1016/j.jrp.2009.08.007

Leyens, J., Paladino, P. M., Rodriguez-Torres, R., Vaes, J., Demoulin, S., Rodriguez-Perez, A. \& Gaunt, R. (2000). The emotional side 
of prejudice: The attribution of secondary emotions to ingroups and outgroups. Personality and Social Psychology Review, 4, 186-197. http://doi .org/10.1207/S15327957PSPR0402_06

Martinovic, B. \& Verkuyten, M. (2013). "We were here first, so we determine the rules of the game": Autochthony and prejudice towards out-groups. European Journal of Social Psychology, 43, 637-647. http://doi.org/10.1002/ejsp.1980

Muratori, M., Delfino, G. I., \& Zubieta E. (2012). SDO and values. Comparing civilian and military students. The 35th Annual Meetings of the International Society of Political Psychology, Chicago.

Pérez-Gil, J.A., Chacón Moscoso, S. \& Moreno Rodríguez, R. (2000) Validez de constructo: el uso del análisis factorial exploratorioconfirmatorio para obtener evidencias de validez. Psicothema, 12(2), 442-446.

Perry, R., Sibley, C. G. \& Duckitt, J. (2013). Dangerous and competitive worldviews: A meta-analysis of their associations with social dominance orientation and right-wing authoritarianism. Journal of Research in Personality, 47, 116-127. http://doi.org/10.1016/j. jrp.2012.10.004

Petit, L. \& Costa, G. (2011). Dominancia social: el género como jerarquía social. Hologramática, 14(3), 71-83.

Pinker, S. (2011). The better angels of our nature: Why violence has declined. New York, NY: Viking.

Pozzo, E. (29 de mayo de 2018). La victoria del activismo feminista en la Argentina. The New York Times. Recuperado de: https:/www.nytimes.com/es/2018/05/29/ opinion-pozzo-debate-aborto-argentina-activismo-feminista/

Pratto F., Liu J., Levin S., Sidanius J., Shih M., Bachrach H. \& Hegarty P. (2000). Social dominance orientation and the legitimization of inequality across cultures. Journal of Cross-Cultural Psychology, 31(3), 369-409. https://doi. org/10.1177/0022022100031003005 
Pratto, F., Sidanius, J. \& Levin, S. (2006). Social dominance theory and the dynamics of intergroup relations: Taking stock and looking forward. European Review of Social Psychology, 17(1), 271-320. https://doi.org/10.1080/10463280601055772

Pratto, F., Sidanius, J., Stallworth, L. \& Malle, B. (1994). Social dominance orientation: A personality variable predicting social and political attitudes. Journal of Personality and Social Psychology, 67(4), 741-763. http://doi.org/10.1037/0022-3514.67.4.741

Pratto, F., Stallworth, L. \& Sidanius, J. (1997). The gender gap: Differences in political attitudes and social dominance orientation. British Journal of Social Psychology, 36, 49-68. https://doi.org/10.1111/j.2044-8309.1997.tb01118.x

Pratto, F., Stewart, A., Foels, R., Henkel, K., Bou Zeineddine, F., \& Morselli, D. (2012, July). Beyond Me and Mine: Low Social Dominance Orientation and Inclusiveness. The 35th Annual Meetings of the International Society of Political Psychology, Chicago.

Quist, R. M. \& Resendez, M. G. (2010). Social dominance threat: Examining social dominance theory's explanation of prejudice as legitimizing myths. Basic and Applied Social Psychology, 24, 287-293. https://doi.org/10.1207/S15324834BASP2404_4

Sibley, C. G., \& Duckitt, J. (2010). The personality bases of ideology: A one-year longitudinal study. Journal of Social Psychology, 150, 540-559. https://doi.org/10.1080/00224540903365364

Sibley, C. G., Harding, J. F., Perry, R., Asbrock, F. \& Duckitt, J. (2010). Personality and prejudice: Extension to the HEXACO personality model. European Journal of Personality, 24, 515-534. http://doi.org/10 .1002/per.750

Sidanius, J., Levin, S., Liu, J. H. \& Pratto, F. (2000) Social dominance orientation, anti-egalitarianism and the political psychology of gender: an extension and cross-cultural replication. European Journal of Social Psychology, 30, 41-67. https:// doi.org/10.1002/(SICI)1099-0992(200001/02)30:1<41: :AID-EJSP976>3.0.CO;2-O 
Sidanius, J., Liu, K., Pratto, F. \& Shaw, J. (1994). Social Dominance Orientation, Hierarchy Attenuators and Hierarchy Enhancers: Social Dominance Theory and the Criminal Justice System. Journal of Applied Social Psychology 24, 338-366. https://doi. org/10.1111/j.1559-1816.1994.tb00586.x

Sidanius, J. \& Pratto, F. (1999). Social Dominance: An Intergroup Theory of Social Hierarchy and Oppression. Cambridge: University Press. Sidanius, J. \& Pratto, F. (2004). Social Dominance Theory: A new synthesis. En Jost, J. y Sidanius, J. (Ed.), Political psychology: Keyreadings (pp.315-332). Nueva York, NY, EE.UU.:Psychology Press. https://doi.org/10.1017/CBO9781139175043.002

Sidanius, J., Pratto, F. \& Bobo, L. (1994). Social dominance orientation and the political psychology of gender: A case of invariance? Journal of Personality and Social Psychology, 67, 998-1011. https://doi.org/10.1037/0022-3514.67.6.998

Sidanius, J., Pratto, F. \& Bobo, L. (1996). Racism, conservatism, affirmative action and intellectual sophistication: A matter of principled conservatism or group dominance? Journal of Personality and Social Psychology, 70, 476-490. https://doi. org/10.1037/0022-3514.70.3.476

Sidanius J., Pratto, F. \& Brief, D. (1995) Group Dominance and the Political Psychology of Gender: A Cross-Cultural Comparison. Political Psychology, 16, 381-396. https://doi. org/10.2307/3791836

Sidanius, J., Pratto, F., Martin, M. \& Stallworth, L. M. (1991). Consensual racism and career track: Some implications of social dominance theory. Political Psychology, 12, 691-721. https://doi. org/10.2307/3791552

Sidanius, J., Pratto, F., Van Laar, C. \& Levin, S. (2004). The Social DominanceTheory: Itsagendaand method. PoliticalPsychology, 25, 845-800. https://doi.org/10.1111/j.1467-9221.2004.00401.x

Sidanius, J., Cotterill, S., Sheehy-Skeffington, J., Kteily, N. \& Carvacho, H. (2016). Social dominance theory: Explorations in the psychology of oppression. En C. Sibley \& F. Barlow 
(Eds.), The Cambridge Handbook of the Psychology of Prejudice (pp. 149-187). Cambridge, Reino Unido: Cambridge University Press. https://doi.org/10.1017/9781316161579.008

Silván-Ferrero, M. P. \& Bustillos, A. (2007). Adaptación de la Escala de Orientación a la Dominancia Social al castellano: validación de la Dominancia Grupal y la Oposición a la Igualdad como factores subyacentes. Revista Psicología Social, 22, 3-15.

Szretter-Noste, E. (2013). Apunte de regresión lineal. Buenos Aires: Universidad de Buenos Aires. Recuperado de: http://mate. dm.uba.ar el día 10 de mayo del 2018.

Thomsen, L., Green, E. G. \& Sidanius, J. (2008). We will hunt them down: How social dominance orientation and rightwing authoritarianism fuel ethnic persecution of immigrants in fundamentally different ways. Journal of Experimental Social Psychology, 44, 1455-1464. http://doi.org/10.1016/j. jesp.2008.06.011

Unger, R. K. (1979). Female and male: Psychological perspectives. New York: Harper \& Row.

Zakrisson, I. (2005). Construction of a short version of the Right Wing Authoritarianism (RWA) scale. Personality and Individual Differences, 39, 863-872. https://doi.org/10.1016/j. paid.2005.02.026

Zubieta, E., Beramendi, M., Sosa, F., \& Torres, A. (2011). Sexismo ambivalente, estereotipos y valores en el ámbito militar. Revista de Psicología, 29(1), 101-130.

Zubieta, E., Delfino, G., \& Fernández, O. (2007). Dominancia social, valores y posicionamiento ideológico en jóvenes universitarios. Psicodebate, 8, 151-169. https://doi.org/10.18682/pd.v8i0.423

Recibido: 19 de febrero, 2020 Revisado: 28 de noviembre, 2020 Aceptado: 3 de marzo, 2021 